\title{
Metric of the universe \\ Emergent gravity from a contracting universe
}

\author{
Sudesh Kumar Arora
}

September 19, 2020

\begin{abstract}
A new metric is derived using cosmological principle, employing the same method as adopted by many authors but by paying closer attention to few hidden assumptions. When solved in weak field limit for space-time around a central mass, the metric gives Newton's gravitational equation. The complete relativistic equation for gravitational acceleration is little different from the one derived using Schwarzschild metric, still all classical GR phenomena such as perihelion precession of Mercury and bending of star light near the Sun are explained. It is argued that spatial curvature is not absolute but rather depends on the frame of reference. Using this new insight, rotational velocity of a star as a function of galactic mass and distance from the galactic center is calculated. It matches MOND equation at sufficient large distances, reduces to Newtonian equations at close range and charts a non-linear wave function in-between. Key observations of cosmology are discussed and it is shown that new model can explain all those with same accuracy or better than the current model without employing any unknowns like dark matter or dark energy.
\end{abstract}

Keywords General relativity · Cosmology · FLRW metric · Schwarzschild metric $\cdot$ MOND

\section{Motivation}

Two metrics are used mainly in general relativity to describe most of the observable universe. At cosmological scales, FLRW metric is used to derive equations for cosmic redshift, age of universe and speed at which galaxies are supposedly moving away from each other (Hubble parameter), while at scales much smaller, the Schwarzschild Metric is used to calculate motion inside gravitational systems like our solar system and all galaxies. Both these

Sudesh Kumar Arora

Bangalore

E-mail: sudekuma@gmail.com 
metrics are successful in explaining most gravity related phenomena except the anomalous velocity of stars orbiting the galaxies, the mechanism behind accelerated expansion of the universe and formation of large scale structures in the universe. Unknown dark energy and dark matter are believed to cause these phenomena but all attempts to find these unknown entities have failed so far. Also tension between measured values of Hubble constant using two different methods is confirmed recently posing a challenge to the $\Lambda$ CDM model.

There are few conceptual problems as well with the two metrics. First is the boundary at which the metric representing the space-time continuum of the universe changes from Schwarzschild metric (non expanding and static space-time) to FLRW metric (expanding space). Einstein and Straus proposed a model with a massive point particle surrounded by a spherically symmetric vacuum region (vacuole) embedded in FLRW universe [12]. The boundary of Einstein-Straus vacuole is an expanding two sphere with it's center being at rest with respect to the Hubble flow. But the Einstein-Straus model demands a very strict spherical symmetry [28]. Hence, its suitability for solving the interplay between cosmic expansion and local physics is doubtful [21]. This model also cannot provide realistic model for relatively smaller structures in our universe. For a single solar mass the vacuole radius is almost 400 light years which is two orders of magnitude larger than the average distance between the stars in our galaxy.

Second problem is that despite both metrics primarily being description of empty space and time, have completely different assumptions about vacuum. The starting point for defining Schwarzschild metric is the assumption that Ricci tensor and stress energy tensor are zero in vacuum. It is assumed that the despite stress-energy tensor being zero, it still somehow gives rise to space-time curvature in the vacuum. This is contrary to basic premise of general relativity that space-time curvature is proportional to the stress energy tensor. FLRW metric on the other hand is constructed assuming Ricci tensor and stressenergy tensor are non-zero in the vast empty spaces between galaxies. This (different assumptions about vacuum ) is a cause of friction between the two models which cannot be reconciled and forms the motivation for current work. To find a unified metric for the universe which treats vacuum consistently and is able to explain gravity related phenomena at all scales.

\section{The metric}

Our universe has 4 dimensions of space-time with maximally symmetric subspace (of 3 dimensional space). The metric of our universe must be of the form as given below [39]

$$
d s^{2}=-c^{2} F(t) d t^{2}+G(t)\left(\frac{d r^{2}}{1-k r^{2}}+r^{2} d \theta^{2}+r^{2} \sin ^{2}(\theta) d \psi^{2}\right)
$$

Here k is the spatial curvature, with $F$ and $G$ being functions of coordinate time $t$ alone, which may or may not be related to each other. We can define 
a scale factor $a$ as $a(t)=\sqrt{G(t)}$ to represent the change in spatial distances, giving us a metric which looks like

$$
d s^{2}=-c^{2} F(t) d t^{2}+a^{2}(t)\left(\frac{d r^{2}}{1-k r^{2}}+r^{2} d \theta^{2}+r^{2} \sin ^{2}(\theta) d \psi^{2}\right)
$$

One can see that by putting $F=1$ above we get FLRW metric, and in fact some authors have actually taken the argument while deriving the FLRW metric that since four velocity is one (in units where $c=1$ ), value of $F(t)$ must also be one [22]. Another and most common approach in literature to derive FLRW metric has been to relabel the coordinate time. Basically a coordinate transformation $t^{\prime}=\int \sqrt{F(t)} d t$ and then dropping the prime gives the FLRW metric $[22,39,38]$.

But there are few problems with both these approaches.

1. It can be shown that for a co-moving observer the magnitude of four velocity is always $c^{2}$ irrespective of the value of metric components $g_{\alpha \beta}$. For example even for Schwarzschild metric the magnitude of four velocity for a co moving observer is $c^{2}$ but clearly $g_{00} \neq 1$. So we cannot simply assume $F=1$.

2. Not all transformations preserve the physical properties of the metric. Any re-labeling which transforms the metric in such a way that the new metric has same dependence on point $P^{\prime}$ (with coordinate $y$ ) as the old metric on another point $P$ (with same value of old coordinate $x=y$ ) satisfies the equation below.

$$
g_{\mu \nu}^{\prime}(y)=g_{\mu \nu}(y)
$$

Only those coordinate transformations (called isometry) which satisfy the equation above preserve the metric and others don't. Now the metric components transforms under change of coordinates $x \rightarrow y$ as

$$
g_{\mu \nu}^{\prime}(y)=\frac{\partial x^{\rho}}{\partial x^{\prime \mu}} \frac{\partial x^{\sigma}}{\partial x^{\prime \nu}} g_{\rho \sigma}(x)
$$

This means that $g_{00}^{\prime}\left(t^{\prime}, r, \theta, \phi\right)=1$. But we can see that $g_{00}\left(t^{\prime}, r, \theta, \phi\right)=$ $F\left(t^{\prime}\right)$ which is not same as $g_{00}^{\prime}\left(t^{\prime}, r, \theta, \phi\right)$ for any value of $t^{\prime}$, meaning the transformation $t^{\prime}=\int \sqrt{F(t)} d t$ is not an isometry.

3. Finally it is clear that rate of proper time in metric 2 changes as a function of time itself, it's not constant. But what about FLRW metric? There is nothing in published literature that mentions a varying rate of proper time in FLRW metric or it's implications. And if rate of proper time does not vary in FLRW metric then it has be physically different than metric 2 since proper time is an invariant.

It is thus established that metric given by 2 is physically different from FLRW metric although both are based on Cosmological principle. We just need to define a value of function $\mathrm{F}$ to get a complete definition. Any non-static value of $\mathrm{F}$ means varying rate of proper time, and also a varying measured 
speed of light as well, unless all scales of length are also varying inversely. What it means is that if time is running faster and faster the speed of light would seem to slow down continuously unless lengths of all measuring rods is also decreasing in same ratio. As it has been confirmed to a high degree of accuracy that speed of light doesn't change with time [3] we can take any possible non-static value for $\mathrm{F}$ provided we accept that size of all atoms and thereby all measuring rods also changes by $1 / \sqrt{F}$.

As scale factor $a$ signifies the change in all spatial distances and size of an atom is nothing but spatial distance between it's outermost electron and it's nucleus, it is the most natural value to assume for change in size of atoms with time. We thus get relationship between $F$ and $a$ as $\sqrt{F}=1 / a(t)$ leading to the complete definition of new metric as

$$
d s^{2}=-\frac{c^{2}}{a^{2}(t)} d t^{2}+a^{2}(t)\left(\frac{d r^{2}}{1-k r^{2}}+r^{2} d \theta^{2}+r^{2} \sin ^{2}(\theta) d \psi^{2}\right)
$$

\section{Emergent gravity from a contracting and non-homogeneous universe}

The new metric is derived above using the cosmological principle, but the universe is not really homogeneous and isotropic as stated by cosmological principle especially at scales of galaxy clusters and below. If we assume scale factor $a(t)$ to be a function of mass density locally, regions of space having higher (or lower) mass density will tend to contract (or expand) faster. This gradient (in tendency to expand or contract) would act like a pseudo force. Assuming for a moment that universe is contracting at all scales then this pseudo force will try to push everything together and the magnitude of this force will directly depend on the mass density. This pseudo force cause by the contracting universe and a non-homogeneous distribution of mass is really the gravitational force.

Even though different regions of universe will have a tendency to contract differently due to mass density variations, the scale factor $a(t)$ may still change at a uniform rate across the universe if there is a counter balancing force to gravity which stops denser regions to become even more denser at a rate higher than other regions. Now centripetal force due to orbital motion of objects in space does counter the gravitational force and stops the denser regions to become more denser at a higher rate. To understand the whole idea better imagine a balloon with raised dots of different sizes on it's surface. Curvature on the surface of each dot is different from each other and more than the surface of balloon but still all the dots and surface of balloon expand or contract at same rate. 


\section{Recovering gravitational equation}

We discussed how gravity is emergent from a contracting and non-homogeneous universe. Now I derive the gravitational equations using the new metric to prove the above hand waving argument. The equation must reduce to Newtonian equation at week field limits and should be able to explain all gravity related phenomena as is done using Schwarzschild solution currently. To achieve this I first calculate the values for Einstein and stress energy tensors and use the same to solve Einstein Field Equations (EFEs). Spatial curvature is assumed to be negligible in week field limits $(\mathrm{k}=0)$ giving us a set of equations

$$
\begin{gathered}
\frac{\hat{a}^{2}}{a^{2}}=-\frac{8 \pi G}{3 a^{2}} \rho \\
a \hat{\hat{a}}+\hat{a}^{2}=4 \pi G\left(\frac{p}{c^{2}}+\frac{\rho}{3}\right) \\
a \hat{\hat{a}}=4 \pi G\left(\frac{p}{c^{2}}+\rho\right)
\end{gathered}
$$

The geodesic equations are solved next

$$
\begin{gathered}
\ddot{t}=\hat{a} a\left(\frac{\dot{t}^{2}}{a^{2}}-\frac{a^{2}}{c^{2}} \dot{r}^{2}-\frac{a^{2}}{c^{2}} r^{2} \dot{\theta}^{2}-\frac{a^{2}}{c^{2}} r^{2} \sin \theta^{2} \dot{\phi}^{2}\right)=-a \hat{a} \\
\ddot{r} a+2 \dot{r} \dot{t} \hat{a}=a r \dot{\theta}^{2}+a r \sin \theta^{2} \dot{\phi}^{2}
\end{gathered}
$$

Now the proper distance between any two celestial objects is product of co-moving distance $r$ and the scale factor $a(t)$

$$
q=r . a(t)
$$

Taking derivative with respect to proper time we get proper (radial) velocity between the two bodies as

$$
\dot{r}^{2} a^{2}=\dot{q}^{2}-r^{2} \dot{a}^{2}-2 q \dot{r} \dot{a}
$$

Here single dot on top denotes first derivative and double dot means second derivative w.r.t. proper time. Using the above in the equation for new metric we get

$$
d s^{2}=-\frac{c^{2}}{a^{2}(t)} d t^{2}+a^{2}(t)\left(d r^{2}+r^{2} d \theta^{2}+r^{2} \sin ^{2}(\theta) d \psi^{2}\right)
$$

Rearranging and using definition of the peculiar velocity $v=\sqrt{\dot{q}^{2}+q^{2} \dot{\theta}^{2}+q^{2} \sin ^{2}(\theta) \dot{\psi}^{2}}$ we get

$$
c^{2}=-\frac{c^{2}}{a^{2}} \dot{t}^{2}+v^{2}-\dot{t}^{2}\left(r^{2} \hat{a}^{2}-2 q \hat{r} \hat{a}\right)
$$


Assuming both the bodies to be co moving with the Hubble flow we can ignore the terms $v$ and $\hat{r}$ giving us

$$
c^{2}=-\dot{t}^{2}\left(\frac{c^{2}}{a^{2}}-r^{2} \hat{a}^{2}\right)
$$

Using 6 the equation above can be written as

$$
c^{2}=-\dot{t}^{2}\left(\frac{c^{2}}{a^{2}}-\frac{8 \pi G \rho q^{2}}{3 a^{2}}\right)
$$

Using formula for mass density $\rho=M / V=3 M / 4 \pi q^{3}$ we get same equation for time dilation as is derived using the Schwarzschild metric which expanded up to first order in Taylor series is

$$
\dot{t}^{2}=-a^{2}-a^{2} \frac{2 G M}{q c^{2}}
$$

Taking derivative of proper velocity with respect to proper time we get proper acceleration between the two bodies as

$$
\ddot{q}=\ddot{r} a+2 \dot{r} \hat{a} \dot{t}+r \hat{\hat{a}} \dot{t}^{2}+r \hat{a} \ddot{t}
$$

Using the equations 9 and 32 and the relation $q=r a$ we get

$$
\ddot{q}=\ddot{r} a+2 \dot{r} \hat{a} \dot{t}-q\left(a \hat{\hat{a}}+\hat{a}^{2}\right)-a \hat{\hat{a}} \frac{2 G M}{c^{2}}
$$

We immediately realize that first two terms on R.H.S are given by 10 and the next two term by 7 and 8 . Replacing we get

$$
\ddot{q}=q \dot{\theta}^{2}+q \sin \theta^{2} \dot{\phi}^{2}-4 \pi G q\left(\frac{p}{c^{2}}+\frac{\rho}{3}\right)-4 \pi G\left(\frac{p}{c^{2}}+\rho\right) \frac{2 G M}{c^{2}}
$$

Again since both the bodies are assumed be co-moving with the Hubble flow $\dot{\theta}=\dot{\phi}=0$ and replacing with the formula for mass density $\rho$ we get in a pressure less situation $(\mathrm{p}=0)$

$$
\ddot{q}=-\frac{G M}{q^{2}}-\frac{6 G^{2} M^{2}}{q^{3} c^{2}}
$$

Immediately we identify first term on the R.H.S. as the Newtonian formula for gravitational acceleration while second term is relativistic and is significant only for large masses and at close distances. 


\section{Recovering MOND}

I have made two assumptions while deriving the equation for gravity. That objects are co-moving with the Hubble flow (no peculiar motion), and that there is no spatial curvature. Now we will discuss a more generic situation where the celestial objects are orbiting a central mass and the spatial curvature is non zero. General relativity posits that space devoid of all matter is flat while geometry of space is spheric in presence of matter, i.e. spatial curvature is positive. But is this spatial curvature absolute, i.e. do all objects feel the crush of this positive spatial curvature irrespective of their state of rest or motion? We know that a stationary object gets pulled towards the central mass while an object which is orbiting the central mass does not. We know the outward centripetal force due to orbital motion cancels the inward gravitational force but what cancels the inward crush due to positive spatial curvature? Should not some mechanism cancel this also for a stable orbit? Just like equal and opposite centripetal acceleration cancels the gravitational acceleration, the positive spatial curvature created by presence of central mass must also be canceled by the equal but negative spatial curvature for a particle in orbital motion. Going by this reasoning we must use a positive value of $\mathrm{k}$ when solving Einstein Field Equations as those represent the positive spatial curvature caused by stress-energy tensor, while for geodesic calculations we must use negative value of $\mathrm{k}$ as that represents the orbital motion of the particle.

New metric with a positive value of $\mathrm{k}$ (not normalized to 1 ) can be written in curvature-normalized coordinates as

$$
d s 2=-\frac{c^{2} d t^{2}}{a^{2}}+a^{2} d r^{2}+a^{2} \frac{\sin ^{2}(r \sqrt{k})}{k} d \theta^{2}+a^{2} \frac{\sin ^{2}(r \sqrt{k})}{k} \sin ^{2} \theta d \phi^{2}
$$

Solving Einstein field equations after simplification gives us 3 equations

$$
\begin{gathered}
\hat{a}^{2}=-8 \pi G \rho-\frac{c^{2} k}{a^{2}} \\
a \hat{\hat{a}}+\hat{a}^{2}=4 \pi G\left(\frac{p}{c^{2}}+\frac{\rho}{3}\right) \\
a \hat{\hat{a}}=4 \pi G\left(\frac{p}{c^{2}}+\rho\right)+\frac{c^{2} k}{a^{2}}
\end{gathered}
$$

Using the formula for density $\rho=M / V$ (where $\mathrm{V}$ is the volume of positively curved 2 sphere) equation 24 becomes

$$
a \hat{\hat{a}}+\hat{a}^{2}=\frac{4 \pi G p}{c^{2}}+\frac{2 G M k^{\frac{3}{2}}}{3 a^{2}(r \sqrt{k}-\sin (r \sqrt{k}) \cos (r \sqrt{k}))}
$$

Similarly 25 becomes

$$
a \hat{\hat{a}}=\frac{4 \pi G p}{c^{2}}+\frac{2 G M k^{\frac{3}{2}}}{a^{2}(r \sqrt{k}-\sin (r \sqrt{k}) \cos (r \sqrt{k}))}+\frac{c^{2} k}{a^{2}}
$$


Next I calculate geodesics for radial $r$ as (using hyperbolic functions as spatial curvature $k^{\prime}<0$ )

$$
\ddot{r} a+2 \dot{r} \dot{a} \dot{t}=\frac{\cosh \left(r \sqrt{\left\|k^{\prime}\right\|}\right)}{\sqrt{\left\|k^{\prime}\right\|}}\left(\sinh \left(r \sqrt{\left\|k^{\prime}\right\|}\right) \dot{\theta}^{2}+\sinh \left(r \sqrt{\left\|k^{\prime}\right\|}\right) \sin ^{2} \theta \dot{\phi}^{2}\right)
$$

As discussed earlier the magnitude of negative spatial curvature due to centripetal motion must be same as positive curvature due to presence of matter, thus $\left\|k^{\prime}\right\|=k$.

$$
\ddot{r} a+2 \dot{r} \dot{a} \dot{t}=\frac{\cosh (r \sqrt{k})}{\sqrt{k}}\left(\sinh (r \sqrt{k}) \dot{\theta}^{2}+\sinh (r \sqrt{k}) \sin ^{2} \theta \dot{\phi}^{2}\right)
$$

Next for time coordinate

$$
\ddot{t}=\hat{a} a\left(\frac{\dot{t}^{2}}{a^{2}}-\frac{a^{2}}{c^{2}} \dot{r}^{2}-\frac{a^{2}}{c^{2}} \sin (r \sqrt{k})^{2} \dot{\theta}^{2}-\frac{a^{2}}{c^{2}} \sin (r \sqrt{k})^{2} \sin (\theta)^{2} \dot{\phi}^{2}\right)=-\hat{a} a
$$

Following the same approach as earlier we have

$$
c^{2}=-\frac{c^{2}}{a^{2}} \dot{t}^{2}+v^{2}-\dot{t}^{2}\left(r^{2} \hat{a}^{2}-2 q \hat{r} \hat{a}\right)
$$

For celestial objects orbiting the central mass in a nearly circular orbit $v<<c$ and $\hat{r}=0$ giving us again

$$
\dot{t}^{2}=-a^{2}-a^{2} \frac{2 G M}{q c^{2}}
$$

We get by using the above in acceleration equation 18

$$
\ddot{q}=v_{o}^{2} \frac{\cosh (r \sqrt{k}) \sqrt{k}}{a^{2} \sinh (r \sqrt{k})}-q\left(a \hat{\hat{a}}+\hat{a}^{2}\right)-\frac{2 G M a \hat{\hat{a}}}{c^{2}}
$$

Here $\mathrm{v}$ is the orbital velocity given as (assuming circular orbit $\Rightarrow \dot{r}=0$ ).

$$
v^{2}=\frac{a^{2} \sinh ^{2}(r \sqrt{k}) \dot{\theta}^{2}}{k}+\frac{a^{2} \sinh ^{2}(r \sqrt{k}) \sin ^{2} \theta \dot{\phi}^{2}}{k}
$$

Using 25 and 26 we get

$$
\ddot{q}=v_{o}^{2} A-B-C-D
$$

Where A,B,C and D are defined as below

$$
\begin{gathered}
A=\frac{\cosh (r \sqrt{k}) \sqrt{k}}{a^{2} \sinh (r \sqrt{k})} \\
B=\frac{2 q G M k^{\frac{3}{2}}}{3 a^{2}(r \sqrt{k}-\sin (r \sqrt{k}) \cos (r \sqrt{k}))}
\end{gathered}
$$




$$
\begin{gathered}
C=\frac{4 G^{2} M^{2} k^{\frac{3}{2}}}{c^{2} a^{2}(r \sqrt{k}-\sin (r \sqrt{k}) \cos (r \sqrt{k}))}+\frac{2 G M k}{a^{2}} \\
D=\frac{4 q \pi G p}{c^{2}}+\frac{8 \pi G^{2} M p}{c^{4}}
\end{gathered}
$$

In a virialized system the particle does not experience any radial acceleration $(\ddot{q}=0)$. Thus we get a formula for virial velocity as

$$
v^{2}=\frac{B+C+D}{A}
$$

It can be shown that in pressure less situation for an object relatively closer to central mass $r^{2}<<1 / k \rightarrow A=1 / q, B / A=G M / q, C / A=6 G^{2} M^{2} / q^{2} c^{2}$ and $D=0$, which means the above equation becomes

$$
v^{2}=-\frac{G M}{q}-\frac{6 G^{2} M^{2}}{q^{2} c^{2}}
$$

On the other hand for in pressure less situation for an object much far away from the central mass $r^{2}>>1 / k, A=\sqrt{k}, B / A=G M \sqrt{k} / 1.5, C / A \approx$ $2 G M \sqrt{k} / a^{2}$ and $D=0$ which means equation 40 reduces to (assuming $\mathrm{a}=1$ )

$$
v^{2}=\frac{8 G M \sqrt{K}}{3}
$$

Putting the value of $k \approx 1 / 4 M$ we recover the MOND equation

$$
v^{2}=\sqrt{G M} \sqrt{\frac{16 G}{9}}=\sqrt{G M a_{0}}
$$

This gives $a_{0}=16 G / 9=1.1864 * 10^{-10}$. This is very close to the value $1.2 * 10^{-10}$ proposed by MOND.

\section{Cosmology}

In this section using the new metric, I show how the size of atoms changes with scale factor and how the energy of photons once emitted does not change. These two factors combined show that universe is contracting at all scales. Next I derive some equations which are used for predicting some of the observed phenomena in the universe at cosmological scales. 
6.1 Change in size of Atoms

I made an assumption while deriving the new metric that that size of atoms is changing with scale factor. I now prove this assumption using the new metric by solving Dirac equation for a hydrogen atom which is given as $[2,24]$

$$
i \gamma^{\mu}\left(\partial \mu-\Gamma_{\mu}-i e A_{\mu}-\frac{m c}{\hbar}\right) \psi=0
$$

Here $\gamma^{\mu}$ are related to $\gamma_{\mu}$ the generalized gamma matrices defining the covariant Clifford algebra $\left\{\gamma_{\mu}, \gamma_{\nu}\right\}=2 g_{\mu \nu}, A_{\mu}$ is the electromagnetic four potential, $e$ is charge of an electron and $\Gamma_{\mu}$ is the spin vector to be determined. As it is convenient to solve Dirac equation in isotropic coordinates I write new metric in isotropic coordinates as

$$
d s^{2}=-\frac{c^{2} d t^{2}}{a^{2}(t)}+\frac{a^{2}(t)}{\left(1+\frac{k r^{2}}{4}\right)^{2}}\left(d x^{2}+d y^{2}+d z^{2}\right)
$$

Dirac equation for the new metric thus becomes after multiplying both sides by $c \gamma^{0}$ and re-arranging

$$
\frac{\left(1+\frac{k r^{2}}{4}\right)}{a(t)} \cdot \gamma^{0} \gamma^{j}\left(\frac{-i \hbar \partial}{\partial x^{j}}\right) \psi(x)=\left(a(t) \cdot \frac{i \hbar c \partial}{\partial x^{0}} \cdot I-\frac{\hbar c z \alpha}{q} \cdot I-m c^{2} \gamma^{0}\right) \psi
$$

Here we have used the relation $\gamma^{0} \cdot \gamma^{0}=I$ and the fact that electromagnetic four potential for simple atoms with spherical symmetry is $A_{0}=\hbar z \alpha / e r$. Where $\alpha$ is the fine structure constant, $z$ is the atomic number and $q$ is the proper distance from the nucleus. Writing the 4 component spinor $\psi$ as 2 two-component spinors

$$
\psi=\left(\begin{array}{l}
\chi \\
\phi
\end{array}\right)
$$

Straightforward simplification and using the quantum mechanic definition of energy and momentum operators, gives a set of two equations

$$
\vec{\sigma} \cdot \vec{p} \phi=-\frac{a(t)}{\left(1+\frac{k r^{2}}{4}\right)}\left(\frac{m c^{2}-E a(t)}{c}+\frac{z \alpha}{q}\right) \chi
$$

and

$$
\vec{\sigma} \cdot \vec{p} \chi=\frac{a(t)}{\left(1+\frac{k r^{2}}{4}\right)}\left(\frac{m c^{2}+E a(t)}{c}-\frac{z \alpha}{q}\right) \phi
$$

Here $\mathrm{q}$ is the proper distance related to co-moving distance $\mathrm{r}$ as

$$
q=\frac{a(t)}{\left(1+\frac{k r^{2}}{4}\right)} r
$$


Defining $m^{\prime}=m \frac{a(t)}{\left(1+\frac{k r^{2}}{4}\right)}$ and $E^{\prime}=E \frac{a^{2}(t)}{\left(1+\frac{k r^{2}}{4}\right)}$ we get the equation in the standard form

$$
\vec{\sigma} \cdot \vec{p} \phi(x)=-\left(\frac{m^{\prime} c^{2}-E^{\prime}}{c}+\frac{z \alpha}{r}\right) \chi(x)
$$

and

$$
\vec{\sigma} \cdot \vec{p} \chi(x)=\left(\frac{m^{\prime} c^{2}+E^{\prime}}{c}-\frac{z \alpha}{r}\right) \phi(x)
$$

Solving these equations (see [10] for detailed calculations), and replacing back values of $m^{\prime}$ and $E^{\prime}$ we get the energy of orbital electron as

$$
E_{n, j}=\frac{m c^{2}}{a(t) \sqrt{1+\frac{Z^{2} \alpha^{2}}{\left(n+\sqrt{\left(j+\frac{1}{2}\right)^{2}-Z^{2} \alpha^{2}}\right)^{2}}}}
$$

As size of atoms is inversely proportional to energy level of its valence electrons, this proves that size of atoms changes in same ratio as the scale factor.

\subsection{Change in frequency of Photons and cosmological redshift}

Equation 53 tells us that energy of photons emitted by a given chemical reaction is a function of time and changes inversely with scale factor $E_{\text {photon }} \propto \frac{1}{a(t)}$. What it means is that energy of photons emitted in a far off galaxy will be lesser or more by a factor of $a(t)$ compared to the ones generated in a lab on Earth using same chemical reaction, provided the energy of the photon does not change once emitted.

Hence a rigorous and appropriate derivation for change in energy of a photon is done by calculating it's geodesic using the metric [9]. The key notion underlying this derivation is the assertion that components of four-momentum of a photon can be defined as $P^{\alpha}=\frac{d x^{\alpha}}{d \lambda}$ where $\lambda$ is a suitable affine parameter. See Appendix B of [39] for the details of the argument. I use the same approach here using the new metric. Since energy-momentum 4-vector of a photon has zero magnitude hence

$$
g_{\mu \nu} P^{\mu} P^{\nu}=-\frac{E^{2}}{a^{2}}+\delta_{i j} a^{2} P^{i} P^{j}=0
$$

This gives us

$$
\delta_{i j} a^{3} P^{i} P^{j}=\frac{E^{2}}{a}
$$

Using the definition of four-momentum of a photon as discussed we get $P^{0}=E / c=d t / d \lambda$ and using the chain rule we can say $d / d \lambda=(d t / d \lambda) \cdot(d / d t)=$ $E / c .(d / d t)$. Zeroth component of the geodesic equation then becomes 


$$
\frac{E}{c} \frac{d E}{d t}=-\Gamma_{00}^{0} \frac{E^{2}}{c^{2}}-\Gamma_{i j}^{0} 0 P^{i} P^{j}=\frac{\dot{a}}{a c^{2}} E^{2}-\delta_{i j} \frac{a^{3} \dot{a}}{c^{2}} P^{i} P^{j}
$$

Using the value of $\delta_{i j} R^{3} P^{i} P^{j}$ calculated above and Christoffel symbols we get

$$
\frac{E}{c} \frac{d E}{d t}=\frac{\dot{a}}{a c^{2}} E^{2}-\frac{\dot{a}}{a c^{2}} E^{2}=0
$$

As E cannot be 0 this means that $d E / d t=0$, i.e. energy of a photon does not change once emitted. As frequency of a photon $\nu$ is a direct function of it's energy $\nu=E / h$ where $h$ is the Planck's constant, it means frequency and wavelength of a photon will also remain unchanged once emitted.

Wavelength is inverse of frequency, and frequency is directly proportional to energy which changes inversely with scale factor as discussed above. This means that if value of scale factor is decreasing with time then wavelength of photons emitted in far off galaxies and received on Earth will be larger compared on the ones generated in a lab on Earth. Thus photons received from distant galaxies must be red-shifted if the universe is contracting at all scales and blue-shifted if it is expanding at all scales. As we observe a red-shift in light coming from far off galaxies, it means that universe is contracting at all scales.

\subsection{Hubble Parameter}

Just like the $\Lambda$ CDM model we can define Hubble parameter as $H=\frac{\hat{a}}{a}$ even though it need not have same value in both models. As discussed spatial curvature $\mathrm{k}$ is inversely proportional to the mass, which mean we can assume its value to be almost zero for the whole universe. Using equation 23 we thus get

$$
H^{2}=-\frac{8 \pi G}{3 a^{2}} \rho
$$

\subsection{Elapsed time}

As $\hat{a}=d a / d t$ we can rewrite the equation 6 as

$$
\left(\frac{d a}{d t}\right)^{2}=-\frac{8 \pi G}{3} \rho
$$

As change in mass-energy density is inversely proportional to cube of scale factor taking current mass-energy density to be $\rho_{0}$ we can rewrite the equation above as

$$
\left(\frac{d a}{d t}\right)^{2}=-\frac{8 \pi G}{3 a^{3}} \rho_{0}
$$


Re arranging we get after taking the square root on both sides

$$
d t=-\frac{a^{\frac{3}{2}} d a}{H_{0}}
$$

Here $H_{0}$ is the current value of Hubble parameter. Integrating both sides of 61 gives us the coordinate time elapsed between the two events. Using relation $1+z=\frac{a}{a_{0}}$ gives us expression for elapsed coordinate time in terms of redshift (assuming $a_{0}=1$ )

$$
\Delta t=\int d t=-\frac{1}{H_{0}} \int(1+z)^{\frac{3}{2}} d z
$$

Proper time is related to coordinate time for a co-moving observer as $d \tau=$ $d t / a(t)$ and hence elapsed proper time can be calculated as

$$
\Delta \tau=-\frac{1}{H_{0}} \int(1+z)^{\frac{1}{2}}
$$

Notice here that rate of coordinate time and proper time are assumed to be same in current epoch (by assuming $R_{0}$ to be 1 ), and proper time was running slower in past compared to today.

\subsection{Distance traveled by light}

Consider a coordinate chart in which a photon is traveling radially towards the observer such that $d \theta=d \psi=0$. The distance traveled by photon will then be

$$
\Delta S=a(t) \int d r
$$

As photons travel along the null geodesic we have, using $d \theta=d \psi=0$

$$
\frac{c^{2}}{a^{2}} d t^{2}=a^{2} d r^{2}
$$

Distance traveled by light then becomes

$$
d=c a(t) \int \frac{1}{a^{2}(t)} d t
$$

Using the relation 62 derived above we get distance traveled by light in terms of redshift

$$
d=-\frac{c(1+z)}{H_{0}} \int_{z}^{0}(1+z)^{-\frac{1}{2}} d z=\frac{2 c(1+z)}{H_{0}}\left((1+z)^{\frac{1}{2}}-1\right)
$$




\subsection{Luminosity Distance}

Luminosity distance $d_{L}$ is a measure of apparent luminosity. There are many factors which impact the measured value of apparent luminosity in addition to the distance between the source and observer (the distance traveled by photon as calculated above)

1. Change in number of photons reaching the observer per unit time Simple calculation shows that interval $\delta t_{0}$ between the successive photons received at the destination is related to interval $\delta t_{1}$ between the successive photons emitted at source as

$$
\delta t_{0}=\delta t_{1} \frac{a\left(t_{0}\right)^{2}}{a\left(t_{1}\right)^{2}}=\frac{\delta t_{1}}{(1+z)^{2}}
$$

A decreasing interval between successive photons means increase in number of photons per unit time reaching the observer, which means decrease in luminosity distance (more luminous objects appear closer)

$$
d_{L 1}=\frac{d_{L}}{(1+z)^{2}}
$$

2. Change in apparent luminosity due to cosmic redshift As wavelength of photons emitted is proportional to the scale factor at that epoch, apparent luminosity of distant objects is reduced by same ratio. This means an increase in luminosity distance. Multiplying above equation with $(1+\mathrm{z})$

$$
d_{L 2}=d_{L 1}(1+z)=\frac{d_{L}}{(1+z)}
$$

3. Change in rate of proper time for the observer

As rate of proper time changes with scale factor all clocks also run faster with time. This means reduction in measured number of photons reaching the observer per unit time and hence and increase in luminosity distance by same factor. Multiplying once more with $(1+z)$

$$
d_{L 3}=d_{L 2}(1+z)=d_{L}
$$

So finally we come to the conclusion that despite multiple factors impacting it, the luminosity distance is simply the distance traveled by the photon as given by 67 .

\subsection{Angular sizes}

For very small angles the angular size of a distant object of linear size L at a distance $\mathrm{d}$ is given as 


$$
\theta=\frac{L}{d}
$$

As the universe is contracting uniformly at all scales, a galaxy of average linear size $L_{s r}$ at current epoch, will be bigger by a factor of $1+z$ at an earlier epoch of time with redshift $z$

$$
L(z)=L_{s r}(1+z)
$$

Using 67, 72 and 73 we get angular size as a function of redshift

$$
\theta(z)=\frac{H_{0} L_{s r}}{2 c\left((1+z)^{\frac{1}{2}}-1\right)}
$$

\section{Predictions and evidence}

\subsection{Perihelion precession of Mercury}

Apsidal angle is the angle between the point of greatest distance of the planet to the point of smallest distance and is given as [16]

$$
\phi=\pi\left[3+q \frac{f^{\prime}(q)}{f(q)}\right]^{\frac{1}{2}}
$$

Where $\mathrm{f}$ is the central force acting on the planet orbiting the sun at the mean radius $q$. Using equation $21 \mathrm{I}$ calculate

$$
q \frac{f^{\prime}(q)}{f(q)}=\frac{\left(2+\frac{18 G M}{c^{2} q^{2}}\right)}{\left(-1-\frac{6 G M}{c^{2} q}\right)}
$$

Using Taylor series approximation to first order we get

$$
q \frac{f^{\prime}(q)}{f(q)} \approx\left(2+\frac{18 G M}{c^{2} q}\right)\left(-1+\frac{6 G M}{c^{2} q}\right) \approx-2\left(1+\frac{3 G M}{c^{2} q}+\ldots\right)
$$

Then precession per orbit is

$$
\delta \phi=2 \phi-2 \pi \approx \frac{6 \pi G M}{c^{2} q}
$$

The value of precession is calculated as 41.11 arc secs per century which is very close to measured value 42.9799 arc secs per century with an error margin of $+-0.0009[36]$. 
7.2 Deflection of light by Sun

Change in velocity of light near a massive object causes it to behave like on optical object with a refractive index given as

$$
n=\frac{c}{c_{r}}
$$

Where $c_{r}$ is the reduced speed of light. The total angle of deflection is the integral along the path of ray of the gradient of $n$ perpendicular to it's path

$$
\alpha=\int \nabla \perp n d l
$$

It can be shown that reduction in speed of light due to gravitational potential is given as

$$
\Delta c_{1}=\frac{G M}{c r}+\frac{3 G^{2} M^{2}}{c^{3} r^{2}}
$$

In addition to this there is reduction in speed of light due to time dilation.

$$
\Delta c_{2}=c-c \frac{d \tau}{d t} \approx \frac{G M}{c r}
$$

Refractive index is then

$$
n=\frac{c}{c_{r}}=\frac{1}{1-\frac{2 G M}{c^{2} r}}
$$

Gradient of Refractive index along the y axis is

$$
\nabla \perp n=\nabla_{y}\left(\frac{1}{1-\frac{2 G M}{c^{2} r}}\right) \approx \frac{2 G M y}{c^{2}\left(x^{2}+y^{2}\right)^{\frac{3}{2}}}
$$

Total angle of deflection is then calculated (at $\mathrm{y}=\mathrm{b}$ ) as

$$
\alpha=\frac{2 G M b}{c^{2}} \int \frac{1}{\left(x^{2}+b^{2}\right)^{\frac{3}{2}}} d x=\frac{2 G M b}{c^{2}}\left[\frac{x}{b^{2} \sqrt{x^{2}+b^{2}}}\right]_{-l_{s} o}^{l_{s} s} \approx \frac{4 G M}{c^{2} b}
$$

Here $l_{s s}$ is the distance of source to sun, $l_{s o}$ is distance of sun to observer, $\mathrm{b}$ is the radius of sun. It is assumed the radius of sun is negligible compared to distance of source and observer from the sun.

$\alpha$ is calculated as 1.751 " which is very close the value measured during the 21 August 2017 total solar eclipse. The final result was a deflection coefficient $\mathrm{L}=1.752$ arcsec, compared to the theoretical value of $\mathrm{L}=1.751$ arcsec, with an uncertainty of only $3 \%$ [4]. 


\subsection{Rotation curves of Galaxies}

Equation 40 when expanded is written as (assuming $\mathrm{a}=1$ )

$$
v^{2}=\frac{2 G M r k \tanh (r \sqrt{k})}{3(r \sqrt{k}-\sin (r \sqrt{k}) \cos (r \sqrt{k}))}
$$

Rotational velocities of stars and gas in outer regions of M31 have been measured to a high degree of certainty out to a distance of $38 \mathrm{Kpc}[5,6,8]$. These measurements show a peculiar shape when plotted on a chart with minimum velocity around $200 \mathrm{~km} / \mathrm{s}$ at a radial distance of around $4-5 \mathrm{Kpc}$, a maximum of $270 \mathrm{Km} / \mathrm{s}$ at around $16-17 \mathrm{Kpc}$, after which the velocity again reduces to a minimum at around $25 \mathrm{Kpc}$ and finally rising to a maximum of $270 \mathrm{~km} / \mathrm{s}$ at $38 \mathrm{Kpc}$ again. If we simply use the Newtonian equation to reverse calculate the central mass needed to generate the measured velocities, it works well till the extended disk (around $16.5 \mathrm{Kpc}$ ) with a cumulative required mass of $\approx 2.9 X 10^{11} M_{\odot}$. This seems realistic as most researchers have estimated the luminous mass of M31 to be in the range of $1-2 X 10^{11} M_{\odot}$ excluding the extended disk. Beyond the extended disk the mass needed to generate the measured velocities becomes unrealistically higher than the measured luminosity.

Assuming that velocities measured till extended disk are mostly due to Newtonian potential and there is negligible contribution from mass density (in line with measured luminosity) in the halo beyond that, I calculate the velocities for all the models. Plotting measured values against the predictions clearly shows Newton's equations predict much lesser orbital velocities beyond the extended disk. MOND also fails to predict the shape of the curve, particularly in the range 16 to $38 \mathrm{Kpc}^{1}$. My equation 40 is the only prediction that really fits not only the magnitude but also the shape of the rotation curve. See figure 1 .

\subsection{Type I-a Supernovae Luminosity versus redshift relation}

Using the relation between distance modulus (difference of apparent and absolute luminosity magnitude $\mu=m-M$ ) to luminosity distance we can compare the prediction of different models with actual observations.

$$
\mu=5 \log _{10}\left(d_{L}\right)+25
$$

Where luminosity distance $d_{L}$ is in Mpc. Using 67 and 87 we get

$$
\mu=5 \log _{10}\left(\frac{2 c}{H_{0}}\right)+5 \log _{10}\left((1+z)\left((1+z)^{\frac{1}{2}}-1\right)\right)+25
$$

1 MOND should be considered only where Newtonian prediction starts to fall below its prediction 
Excellent measurements of high redshift supernovae have been done by many teams independently $[18,26,23,32,30,27,7]$. The SCP "Union2.1" SN Ia compilation [32] is a compilation of many such studies bringing together data for $833 \mathrm{SNe}$, drawn from 19 datasets including data from Noble prize winners Supernova Cosmology team and High Z Supernova team. Of these, 580 SNe pass usability cuts, which I have used as the test set. See figure 2 for comparison of different models.

\subsection{Time dilation}

As per new metric derived in this paper time was actually running slower in past compared to today by a factor of $1+z$. This has been confirmed as increased light curve widths of the supernova [25,43]. A plot of width factor $\mathrm{w}$ versus the redshift data is shown in Figure 3.

\subsection{Angular size evolution}

The angular size as a function of redshift in new model is given by the equation 74. Combining the analysis of FIRES data with the results obtained by GEMS at $z<1$ [1] and tying both to the present-day results from SDSS [29], a detailed luminosity-size relation was derived for a redshift range of $0.2<z<2.5$ [35]. To match the predicted angular sizes it is hypothesized that deficit of larger angular sizes at high redshifts, as predicted in an expanding Universe, is an evolutionary effect by which galaxies were smaller in the past.

Using the factor by which the galaxies are hypothesized to be smaller at a given red shift, I reverse calculate the measured angular sizes of galaxies by multiplying it with the predicted size as per standard model. Comparing this with the predicted values of angular sizes as per new metric derived in this paper and tired light model we find that measured angular sizes perfectly match the predicted sizes. See Figure 4. Standard model prediction on the other hand does not match the observations unless we assume that the size of galaxies was in past somehow smaller for a given mass or luminosity.

\subsection{Fine structure constant}

In the non-relativistic limit, all atomic spectra are proportional to the constant $m e^{4} / \hbar^{2}$ and therefore any analyses of quasar spectra in this limit cannot detect any change in fine structure constant $\alpha$. However any change of $\alpha$ can be found by measuring the change in size of relativistic corrections, as those are proportional to $\alpha^{2}$.

$$
\Delta E_{b} \approx-\frac{m e^{4} Z^{2}}{2 \hbar^{2}} \frac{(Z \alpha)^{2}}{n^{3}}\left(\frac{1}{j+\frac{1}{2}}-\frac{3}{4 n}\right)
$$


Same principle has been used while determining the variation in value of $\alpha$ over cosmological distances [11]. It has been assumed that wavelengths of all photons get stretched by a factor of $1+z$ as those travel through an expanding universe, where $\mathrm{z}$ is the redshift parameter. This implies that spectral lines due to relativistic corrections should also stretch by the same factor if value of $\alpha$ does not vary with time and space. Equations 51 and 52 also show that only change in wavelength of relativistic corrections will be stretching by a factor of $1+z$ if ratio of $\alpha$ and co-moving radius $\mathrm{r}$ of an atom does not change. As no variation in $\alpha$ was measured it means that co moving size of atoms also does not change implying the change in proper size of atoms and molecules.

\subsection{Cosmic microwave background radiation}

The temperature of CMB radiation is highly uniform across the sky with fluctuations of less than 1 part in 100,000. In contrast to this smooth beginning our present day universe is a clumpy place with vast voids and narrow filaments of galaxy clusters. $\Lambda \mathrm{CDM}$ model employs dark matter as the clumping agent to explain this transition. Same dark matter is claimed to be present in the halos of galaxies even today. But in this paper I have been able to explain the virial velocities most accurately using the new metric without employing the dark matter. If there is no dark matter in the universe, it is impossible to explain the formation of large scale structures from a smooth beginning. This means $\mathrm{CMB}$ is the biggest evidence against the $\Lambda \mathrm{CDM}$ model and not for it.

Although this paper does not explain the CMB, couple of alternative explanations for $\mathrm{CMB}$ are already available in literature to explain the source of this radiation. HJ Fahr in his 2009 paper [13] hypothesized CMB as the microwaves generated by photon-photon interactions meditated by electronpositron pair produced spontaneously in the vacuum. He has suggested few tests and observations also to verify or negate his claim. Fahr's hypothesis also explains the observed ratio of photons to matter particles. He also highlighted some problems with CMB being the ancient radiation from the re-combination era in 2015 which raise serious doubts over the currently accepted notion related to CMB [14]. Some association has also been reported between galactic neutral Hydrogen structure and angular distribution of CMB [37]. More work needs to be done in this area to understand the real source of this radiation.

\section{Discussion and conclusion}

The new model proposed in the paper based on a new metric, suggests that universe is contracting uniformly at all scales, rather than expanding as it currently assumed. But this is not a completely new idea. At least three proposals for a contracting universe are already available in literature. First by William Sumner suggests that strength of the electrical force between charges is changing with evolving universe, causing shifts in energy levels of atoms. 
This shift causes change in wavelength of emitted light which is twice as large as shift in wavelength of photons due to evolving universe. Using this logic he concluded that Friedman Universe is contracting rather than expanding. [31]. As per the hypothesis the size of atoms is contracting at twice the speed than the entire universe, which means ratio of inter-galactic distances to the size of atoms is increasing with time just like standard model. Second model proposed by Wetterich, also known as variable gravity model, posits that strength of gravity as measured by Newton's constant or the Planck mass, depends on the value of a scalar field, called Cosmon. With a shrinking scale factor, the masses of particles increase and sizes of atoms shrinks. Picture of a shrinking Universe can be mapped to the usual picture of an expanding Universe by a field transformation of the metric. $[42,41,40]$. In this model also ratio of intergalactic distances to the size of atoms increases with time just as in Sumner model. Finally Traunmuller's proposal called hidden contraction model, suggests that "physical processes speed up and objects contract uniformly as a function of time, standards of measurements not excluded, and only free waves being excepted" [34]. As ratio of galactic distances and atomic sizes does not change in this model, it has the potential to make predictions different from both standard model and steady state model just like my work. But Traunmuller's proposal is purely phenomenological and does not provide any clues to the underlying physics driving the perpetual contraction of space and time. My work on the other hand uses general relativity to build the mathematical model and to explain the underlying physics behind the contraction of universe.

Finally my work suggests some new notions and possible tests to verify my proposal. Spatial curvature being inversely proportional to the mass is opposite of the currently accepted notion that curvature is higher near the massive objects like black holes. This may have significant impact on the way we think about sub atomic particles having very small masses as the spatial curvature at those scales will be very high. Another insight is the magnitude of gravitational force itself in different regimes. Very close to the mass the extra term for gravitational acceleration due to general relativity is two times stronger than the one predicted by Schwarzschild metric (equation 21). At distances between the Newtonian and MOND regimes, the forces is non-linear in nature and is different from both Newtonian and MOND prediction (equation ??). Both these can be verified experimentally to verify the new model proposed in this paper.

\section{References}

1. Barden, M., Rix, H., Somerville, R.S., Bell, E.F., Hausler, B., Peng, C.Y., Borch, A., Beckwith, S.V.W., Caldwell, J.A.R., Heymans, C., Jahnke, K., Jogee, S., McIntosh, D.H., Meisenheimer, K., Sanchez, S.F., Wisotzki, L., Wolf, C.: GEMS: The Surface Brightness and Surface Mass Density Evolution of Disk Galaxies. Astrophys. J. 635(2), 959-981 (2005). DOI 10.1086/497679. URL http://iopscience.iop.org/0004$637 \mathrm{X} / 635 / 2 / 959 /$ fulltext/ 
2. Barut, A.O., Duru, I.H.: Exact solutions of the Dirac equation in spatially flat Robertson-Walker space-times. Phys. Rev. D 36(12), 3705-3711 (1987). DOI 10.1103/PhysRevD.36.3705. URL http://link.aps.org/doi/10.1103/PhysRevD.36.3705

3. Braxmaier, C., Müller, H., Pradl, O., Mlynek, J., Peters, A., Schiller, S.: Tests of relativity using a cryogenic optical resonator. Phys. Rev. Lett. 88(1), 010401 (2002). DOI 10.1103/PhysRevLett.88.010401. URL http://www.ncbi.nlm.nih.gov/pubmed/11800924

4. Bruns, D.G.: Gravitational starlight deflection measurements during the 21 August 2017 total solar eclipse. Class. Quantum Gravity 35(7) (2018). DOI 10.1088/13616382/aaaf2a. URL http://arxiv.org/abs/1802.00343 http://dx.doi.org/10.1088/13616382 /aaaf2a

5. Carignan, C., Chemin, L., Foster, T.: Extended HI rotation curve of M31 using deep DRAO observations. Proc. Int. Astron. Union 2(S235), 193 (2006). DOI 10.1017/S1743921306005990. URL http://arxiv.org/abs/astro-ph/0702609

6. Chemin, L., Carignan, C., Foster, T.: H i Kinematics and dynamics of messier 31. Astrophys. J. 705(2), 1395-1415 (2009). DOI 10.1088/0004-637X/705/2/1395. URL http://arxiv.org/abs/0909.3846

7. Conley, A., Howell, D.A., Howes, A., Sullivan, M., Astier, P., Balam, D., Basa, S., Carlberg, R.G., Fouchez, D., Guy, J., Hook, I., Neill, J.D., Pain, R., Perrett, K., Pritchet, C.J., Regnault, N., Rich, J., Taillet, R., Aubourg, E., Bronder, J., Ellis, R.S., Fabbro, S., Filiol, M., Le Borgne, D., Palanque-Delabrouille, N., Perlmutter, S., Ripoche, P.: The Rise Time of Type Ia Supernovae from the Supernova Legacy Survey. Astron. J. 132(4), 1707-1713 (2006). DOI 10.1086/507788. URL http://stacks.iop.org/1538$3881 / 132 / \mathrm{i}=4 / \mathrm{a}=1707$

8. Corbelli, E., Lorenzoni, S., Walterbos, R., Braun, R., Thilker, D.: A wide-field H I mosaic of Messier 31. Astron. Astrophys. 511, A89 (2010). DOI 10.1051/0004-6361/200913297. URL http://www.aanda.org/10.1051/0004-6361/200913297

9. Dandelson, S.: Modern Cosmology. Academic Press (2003)

10. Darwin, C.G.: The Wave Equations of the Electron. Proc. R. Soc. A Math. Phys. Eng. Sci. 118(780), 654-680 (1928). DOI 10.1098/rspa.1928.0076. URL http://rspa.royalsocietypublishing.org/content/118/780/654.abstract

11. Dzuba, V.A., Flambaum, V.V., Webb, J.K.: Calculations of the relativistic effects in many-electron atoms and space-time variation of fundamental constants. Phys. Rev. A 59(1), 230-237 (1999). DOI 10.1103/PhysRevA.59.230. URL http://link.aps.org/doi/10.1103/PhysRevA.59.230

12. Einstein, A., Straus, E.G.: The Influence of the Expansion of Space on the Gravitation Fields Surrounding the Individual Stars. Rev. Mod. Phys. 17(2-3), 120-124 (1945). DOI 10.1103/RevModPhys.17.120. URL http://link.aps.org/doi/10.1103/RevModPhys.17.120

13. Fahr, H., Zönnchen, J.: The writing on the cosmic wall: Is there a straightforward explanation of the cosmic microwave background? Ann. Phys. 18(10-11), 699-721 (2009). DOI 10.1002/andp.200910365. URL http://doi.wiley.com/10.1002/andp.200910365

14. Fahr, H.J., Sokaliwska, M.: Remaining Problems in Interpretation of the Cosmic Microwave Background. Phys. Res. Int. 2015, 1-15 (2015). DOI 10.1155/2015/503106. URL https://www.hindawi.com/archive/2015/503106/

15. Ferguson, A.M.N., Irwin, M.J., Ibata, R.A., Lewis, G.F., Tanvir, N.R.: Evidence for Stellar Substructure in the Halo and Outer Disk of M31. Astron. J. 124(3), 1452-1463 (2002). DOI 10.1086/342019. URL http://stacks.iop.org/1538-3881/124/i=3/a=1452

16. Fitzpatrick, R.: An Introduction to Celestial Mechanics. Cambridge University Press, Cambridge (2012). DOI 10.1017/CBO9781139152310. URL http://ebooks.cambridge.org/ref/id/CBO9781139152310

17. Goldhaber, G., Groom, D.E., Kim, A., Aldering, G., Astier, P., Conley, A., Deustua, S.E., Ellis, R., Fabbro, S., Fruchter, A.S., Goobar, A., Hook, I., Irwin, M., Kim, M., Knop, R.A., Lidman, C., McMahon, R., Nugent, P.E., Pain, R., Panagia, N., Pennypacker, C.R., Perlmutter, S., RuizLapuente, P., Schaefer, B., Walton, N.A., York, T.: Timescale Stretch Parameterization of Type Ia Supernova B Band Light Curves. Astrophys. J. 558(1), 359-368 (2001). DOI 10.1086/322460. URL http://stacks.iop.org/0004$637 \mathrm{X} / 558 / \mathrm{i}=1 / \mathrm{a}=359$ 
18. Guy, J., Sullivan, M., Conley, A., Regnault, N., Astier, P., Balland, C., Basa, S., Carlberg, R.G., Fouchez, D., Hardin, D., Hook, I.M., Howell, D.A., Pain, R., PalanqueDelabrouille, N., Perrett, K.M., Pritchet, C.J., Rich, J., Ruhlmann-Kleider, V., Balam, D., Baumont, S., Ellis, R.S., Fabbro, S., Fakhouri, H.K., Fourmanoit, N., GonzálezGaitán, S., Graham, M.L., Hsiao, E., Kronborg, T., Lidman, C., Mourao, A.M., Perlmutter, S., Ripoche, P., Suzuki, N., Walker, E.S.: The Supernova Legacy Survey 3 -year sample: Type Ia supernovae photometric distances and cosmological constraints. Astron. Astrophys. 523, A7 (2010). DOI 10.1051/0004-6361/201014468. URL http://www.aanda.org/10.1051/0004-6361/201014468

19. Ibata, R., Martin, N.F., Irwin, M., Chapman, S., Ferguson, A.M.N., Lewis, G.F., McConnachie, A.W.: The Haunted Halos of Andromeda and Triangulum: A Panorama of Galaxy Formation in Action. Astrophys. J. 671(2), 1591-1623 (2007). DOI 10.1086/522574. URL http://stacks.iop.org/0004-637X/671/i=2/a=1591

20. Jones, D.O., Rodney, S.A., Riess, A.G., Mobasher, B., Dahlen, T., McCully, C., Frederiksen, T.F., Casertano, S., Hjorth, J., Keeton, C.R., Koekemoer, A., Strolger, L.G., Wiklind, T.G., Challis, P., Graur, O., Hayden, B., Patel, B., Weiner, B.J., Filippenko, A.V., Garnavich, P., Jha, S.W., Kirshner, R.P., Ferguson, H.C., Grogin, N.A., Kocevski, D.: THE DISCOVERY OF THE MOST DISTANT KNOWN TYPE Ia SUPERNOVA AT REDSHIFT 1.914. Astrophys. J. 768(2), 166 (2013). DOI 10.1088/0004-637X/768/2/166. URL http://stacks.iop.org/0004$637 \mathrm{X} / 768 / \mathrm{i}=2 / \mathrm{a}=166 ? \mathrm{key}=$ crossref. $8303511 \mathrm{~d} 40753 \mathrm{~d} 9714 \mathrm{be}$ bcacfa5ef60

21. Mars, M.: Axially symmetric Einstein-Straus models. Phys. Rev. D 57(6), 3389-3400 (1998). DOI 10.1103/PhysRevD.57.3389. URL http://arxiv.org/abs/gr-qc/0202087

22. Misner, C.W., Thorne, K.S., Wheeler, J.A.: Gravitation, first edn. W H Freeman and Company (1973)

23. Perlmutter, S., Gabi, S., Goldhaber, G., Goobar, A., Groom, D.E., Hook, I.M., Kim A.G., Kim, M.Y., Lee, J.C., Pain, R., Pennypacker, C.R., Small, I.A., Ellis, R.S., McMahon, R.G., Boyle, B.J., Bunclark, P.S., Carter, D., Irwin, M.J., Glazebrook, K., Newberg, H.J.M., Filippenko, A.V., Matheson, T., Dopita, M., Couch, W.J.: Measurements of the Cosmological Parameters $\Omega$ and $\Lambda$ from the First Seven Supernovae at z 0.35. Astrophys. J. 483(2), 565-581 (1997). DOI 10.1086/304265. URL http://stacks.iop.org/0004$637 \mathrm{X} / 483 / \mathrm{i}=2 / \mathrm{a}=565$

24. Pollock, M.D.: ON THE DIRAC EQUATION IN CURVED SPACE-TIME. ACTA Phys. Pol. B 41(8) (2010). URL http://www.actaphys.uj.edu.pl/fulltext?series $=$ Reg\&vol=41\&page $=1827$

25. Riess, A.G., Filippenko, A.V., Leonard, D.C., Schmidt, B.P., Suntzeff, N., Phillips, M.M., Schommer, R., Clocchiatti, A., Kirshner, R.P., Garnavich, P., Challis, P., Leibundgut, B., Spyromilio, J., Smith, R.C.: Time Dilation from Spectral Feature Age Measurements of Type IA Supernovae. Astron. J. 114, 722 (1997). DOI 10.1086/118506. URL http://adsabs.harvard.edu/cgi-bin/bib_query?1997AJ....114..722R

26. Riess, A.G., Strolger, L.g., Tonry, J., Casertano, S., Ferguson, H.C., Mobasher, B., Challis, P., Filippenko, A.V., Jha, S., Li, W., Chornock, R., Kirshner, R.P., Leibundgut, B., Dickinson, M., Livio, M., Giavalisco, M., Steidel, C.C., Benitez, T., Tsvetanov, Z.: Type Ia Supernova Discoveries at $\mathrm{z}>1$ from the Hubble Space Telescope : Evidence for Past Deceleration and Constraints on Dark Energy Evolution. Astrophys. J. 607(2), 665-687 (2004). DOI 10.1086/383612. URL http://stacks.iop.org/0004-637X/607/i=2/a=665

27. Rubin, D., Knop, R.A., Rykoff, E., Aldering, G., Amanullah, R., Barbary, K. Burns, M.S., Conley, A., Connolly, N., Deustua, S., Fadeyev, V., Fakhouri, H.K., Fruchter, A.S., Gibbons, R.A., Goldhaber, G., Goobar, A., Hsiao, E.Y., Huang, X., Kowalski, M., Lidman, C., Meyers, J., Nordin, J., Perlmutter, S., Saunders, C., Spadafora, A.L., Stanishev, V., Suzuki, N., Wang, L.: PRECISION MEASUREMENT OF THE MOST DISTANT SPECTROSCOPICALLY CONFIRMED SUPERNOVA Ia WITH THE HUBBLE SPACE TELESCOPE. Astrophys. J. 763(1), 35 (2013). DOI 10.1088/0004-637X/763/1/35. URL http://stacks.iop.org/0004$637 \mathrm{X} / 763 / \mathrm{i}=1 / \mathrm{a}=35 ? \mathrm{key}=$ crossref.e868c4fbda5e9ee9a5a53dfeb99a09c7

28. Senovilla, J.M.M., Vera, R.: Impossibility of the Cylindrically Symmetric Einstein-Straus Model. Phys. Rev. Lett. 78(12), 2284-2287 (1997). DOI 10.1103/PhysRevLett.78.2284. D http://link.aps.org/doi/10.1103/PhysRevLett.78.2284 
29. Shen, S., Mo, H.J., White, S.D.M., Blanton, M.R., Kauffmann, G., Voges, W., Brinkmann, J., Csabai, I.: The size distribution of galaxies in the Sloan Digital Sky Survey. Mon. Not. R. Astron. Soc. 343(3), 978-994 (2003). DOI 10.1046/j.13658711.2003.06740.x. URL http://mnras.oxfordjournals.org/content/343/3/978

30. Sullivan, M., Guy, J., Conley, A., Regnault, N., Astier, P., Balland, C., Basa, S., Carlberg, R.G., Fouchez, D., Hardin, D., Hook, I.M., Howell, D.A., Pain, R., PalanqueDelabrouille, N., Perrett, K.M., Pritchet, C.J., Rich, J., Ruhlmann-Kleider, V., Balam, D., Baumont, S., Ellis, R.S., Fabbro, S., Fakhouri, H.K., Fourmanoit, N., GonzálezGaitán, S., Graham, M.L., Hudson, M.J., Hsiao, E., Kronborg, T., Lidman, C., Mourao, a.M., Neill, J.D., Perlmutter, S., Ripoche, P., Suzuki, N., Walker, E.S.: SNLS3: CONSTRAINTS ON DARK ENERGY COMBINING THE SUPERNOVA LEGACY SURVEY THREE-YEAR DATA WITH OTHER PROBES. Astrophys. J. 737(2), 102 (2011). DOI 10.1088/0004-637X/737/2/102. URL http://stacks.iop.org/0004$637 \mathrm{X} / 737 / \mathrm{i}=2 / \mathrm{a}=102$ ?key=crossref.e735c12c199d3274597f8a25549eebcd

31. Sumner, W.Q.: On the variation of vacuum permittivity in Friedmann universes. Astrophys. J. 429, 491 (1994). DOI 10.1086/174338. URL http://adsabs.harvard.edu/doi/10.1086/174338

32. Suzuki, N., Rubin, D., Lidman, C., Aldering, G., Amanullah, R., Barbary, K., Barrientos, L.F., Botyanszki, J., Brodwin, M., Connolly, N., Dawson, K.S., Dey, A., Doi, M., Donahue, M., Deustua, S., Eisenhardt, P., Ellingson, E., Faccioli, L., Fadeyev, V., Fakhouri, H.K., Fruchter, A.S., Gilbank, D.G., Gladders, M.D., Goldhaber, G., Gonzalez, A.H., Goobar, A., Gude, A., Hattori, T., Hoekstra, H., Hsiao, E., Huang, X., Ihara, Y., Jee, M.J., Johnston, D., Kashikawa, N., Koester, B., Konishi, K., Kowalski, M., Linder, E.V., Lubin, L., Melbourne, J., Meyers, J., Morokuma, T., Munshi, F., Mullis, C., Oda, T., Panagia, N., Perlmutter, S., Postman, M., Pritchard, T., Rhodes, J., Ripoche, P., Rosati, P., Schlegel, D.J., Spadafora, A., Stanford, S.A., Stanishev, V., Stern, D., Strovink, M., Takanashi, N., Tokita, K., Wagner, M., Wang, L., Yasuda, N., Yee, H.K.C.: THE HUBBLE SPACE TELESCOPE CLUSTER SUPERNOVA SURVEY. V. IMPROVING THE DARK-ENERGY CONSTRAINTS ABOVE $\mathrm{z}>1$ AND BUILDING AN EARLY-TYPE-HOSTED SUPERNOVA SAMPLE. Astrophys. J. 746(1), 85 (2012). DOI 10.1088/0004-637X/746/1/85. URL http://stacks.iop.org/0004$637 \mathrm{X} / 746 / \mathrm{i}=1 / \mathrm{a}=85$ ?key=crossref.4d6837913710c5bf10f23d3e15e98d35

33. Tanaka, M., Chiba, M., Komiyama, Y., Guhathakurta, P., Kalirai, J.S., Iye, M.: STRUCTURE AND POPULATION OF THE ANDROMEDA STELLAR HALO FROM A SUBARU/SUPRIME-CAM SURVEY. Astrophys. J. 708(2), 1168-1203 (2010). DOI 10.1088/0004-637X/708/2/1168. URL http://stacks.iop.org/0004$637 \mathrm{X} / 708 / \mathrm{i}=2 / \mathrm{a}=1168$ ?key=crossref.39ab1acf4534f131eba9f5768f1d8c1d

34. Traunmüller, H.: From magnitudes and redshifts of supernovae, their lightcurves, and angular sizes of galaxies to a tenable cosmology. Astrophys. Space Sci. 350(2), 755-767 (2014). DOI 10.1007/s10509-013-1764-z. URL http://link.springer.com/10.1007/s10509-013-1764-z

35. Trujillo, I., Forster Schreiber, N.M., Rudnick, G., Barden, M., Franx, M., Rix, H. Caldwell, J.A.R., McIntosh, D.H., Toft, S., Haussler, B., Zirm, A., van Dokkum, P.G., Labbe, I., Moorwood, A., Rottgering, H., van der Wel, A., van der Werf, P., van Starkenburg, L.: The Size Evolution of Galaxies since z 3: Combining SDSS, GEMS, and FIRES. Astrophys. J. 650(1), 18-41 (2006). DOI 10.1086/506464. URL http://iopscience.iop.org/0004-637X/650/1/18/fulltext/

36. TURYSHEV, S.G., WILLIAMS, J.G.: Space-based tests of gravity with laser ranging. Int. J. Mod. Phys. D 16(12a), 2165-2179. DOI 10.1142/S0218271807011838. URL http://arxiv.org/abs/gr-qc/0611095 http://dx.doi.org/10.1142/S0218271807011838 http://www.worldscientific.com/doi/abs/10.1142/S0218271807011838

37. Verschuur, G.L.: ON THE APPARENT ASSOCIATIONS BETWEEN INTERSTELLAR NEUTRAL HYDROGEN STRUCTURE AND ( WMAP ) HIGHFREQUENCY CONTINUUM EMISSION. Astrophys. J. 711(2), 1208-1228 (2010). DOI 10.1088/0004-637X/711/2/1208. URL http://stacks.iop.org/0004$637 \mathrm{X} / 711 / \mathrm{i}=2 / \mathrm{a}=1208$ ?key=crossref.efca1e805fdae8e36275b36f95be1a8d

38. Wald, R.M.: General Relativity, 1st editio edn. University Of Chicago Press (1984) 
39. Weinberg, S.: Gravitation and Cosmology: Principles and Applications of the General Theory of Relativity, vol. -1. John WIley \& Sons, Inc. (1972). URL http://adsabs.harvard.edu/abs/1972gcpa.book.....W

40. Wetterich, C.: Universe without expansion Phys. Dark Universe $\quad \mathbf{2}(4), \quad 184-187$ (2013). $\quad$ DOI 10.1016/j.dark.2013.10.002. URL http://linkinghub.elsevier.com/retrieve/pii/S2212686413000332

41. Wetterich, C.: Eternal Universe. Phys. Rev. D 90(4), $043520 \quad$ (2014). DOI 10.1103/PhysRevD.90.043520. URL http://link.aps.org/doi/10.1103/PhysRevD.90.043520

42. Wetterich, C.: Variable gravity Universe. Phys. Rev. D $\mathbf{8 9}(2)$, 024005 (2014). DOI 10.1103/PhysRevD.89.024005. URL http://link.aps.org/doi/10.1103/PhysRevD.89.024005

43. Wilson, O.C.: Possible Applications of Supernovae to the Study of the Nebular Red Shifts. Astrophys. J. 90 (1939). URL http://articles.adsabs.harvard.edu/cgi-bin/nphjournal_query? volume $=90 \&$ plate_select $=$ NO\&page $=634 \&$ plate $=\&$ cover $=\&$ journal $=$ ApJ. 


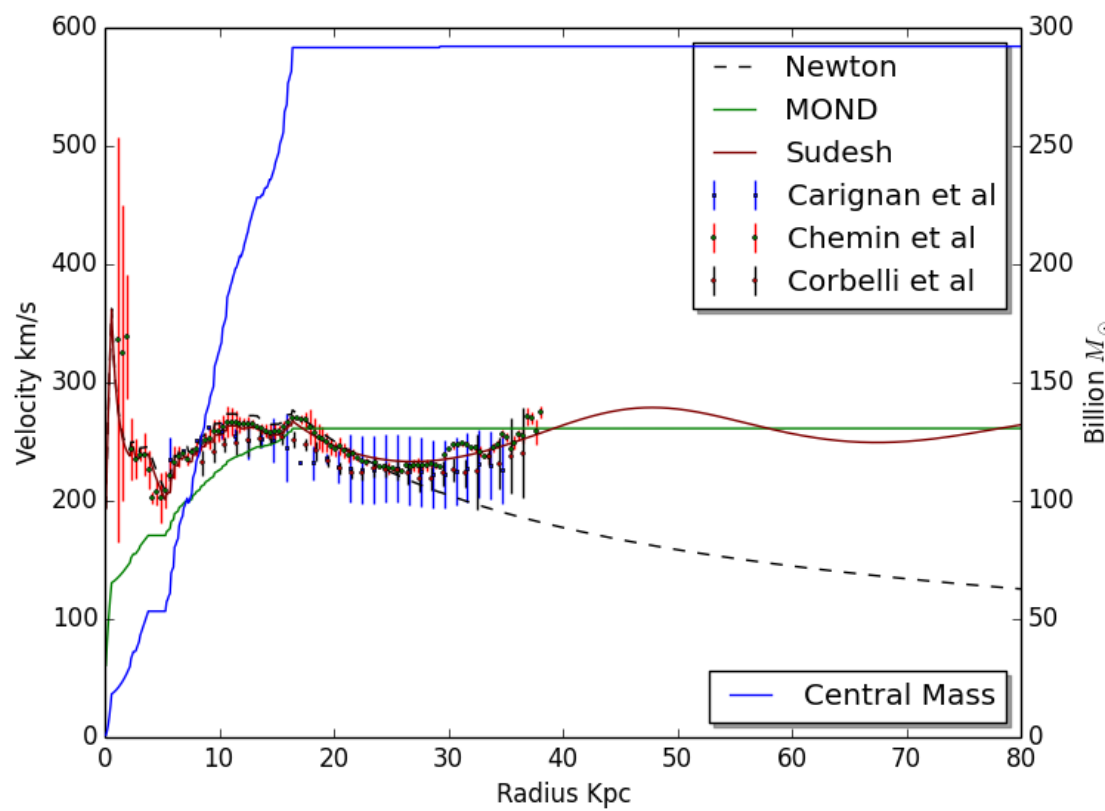

Fig. 1 Rotational velocities in the outer regions of M31. Distance from the center is in Kpc. Central mass is also plotted as a function of radius in blue. Data well beyond the measured velocities has been plotted to show that equation 40 asymptotes to MOND prediction at larger distances. It should not be inferred as the predicted values for rotational velocities as there is more structure beyond $40 \mathrm{Kpc}$ which impacts the rotational velocities at larger distances $[15,19,33]$ 


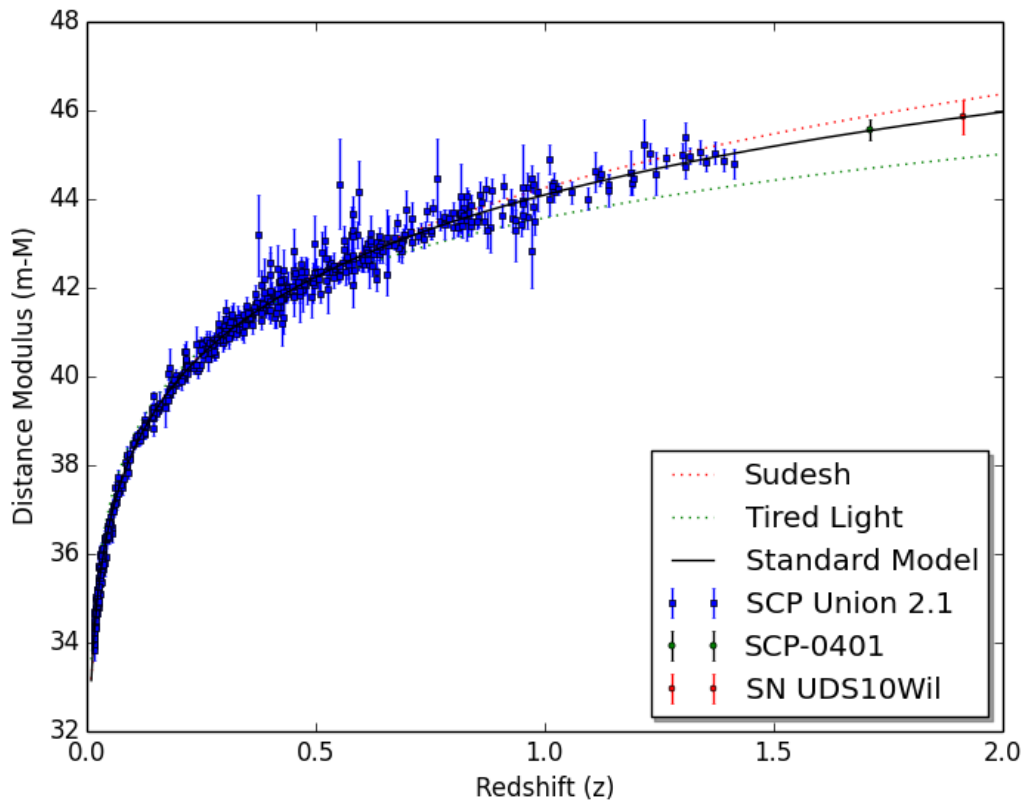

Fig. 2 Distance modulus versus redshift data from SCP Union 2.1 compilation. Two additional data points for highest redshift SNe SCP-0401 [27] and UDS10Wil [20] discovered after the compilation have been added to check the model fit to data at higher $\mathrm{z}$ values. The tired light model is completely ruled out due to poor fit with data 


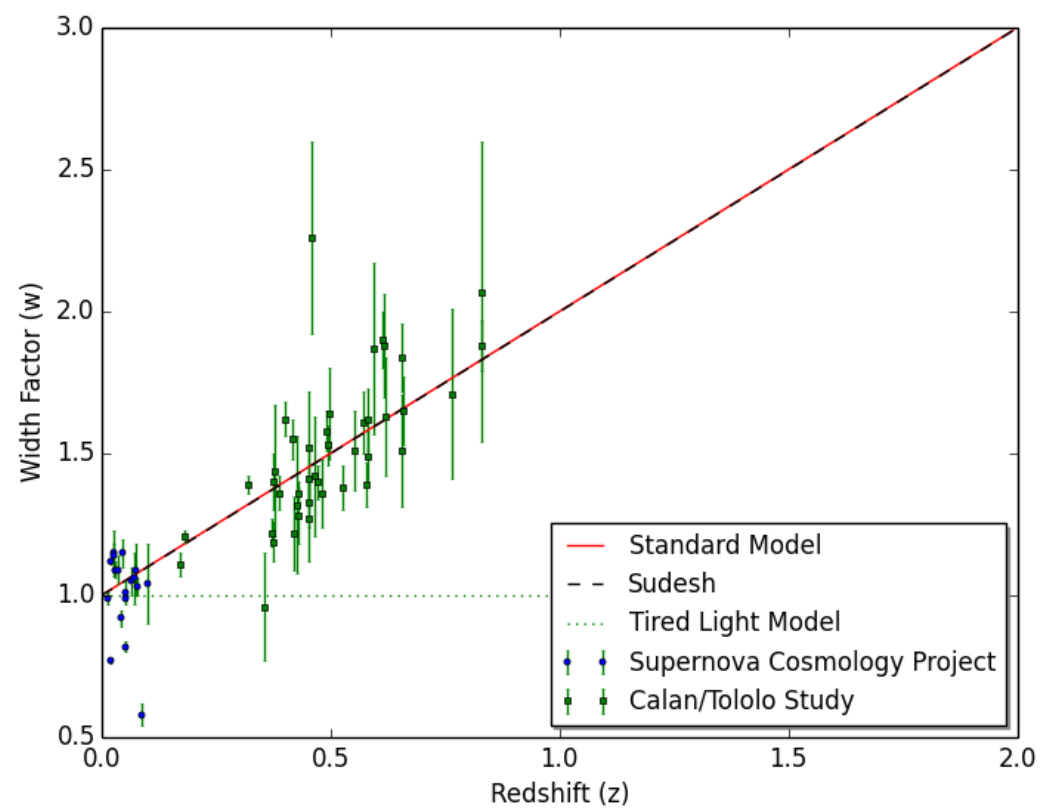

Fig. 3 Variation of width factor of Supernovae versus redshift [17]. Both $\Lambda C D M$ model and my model fit the data well. The tired light model is completely ruled out as it does not predict any time dilation and hence cannot account for widening of Supernova light curves 


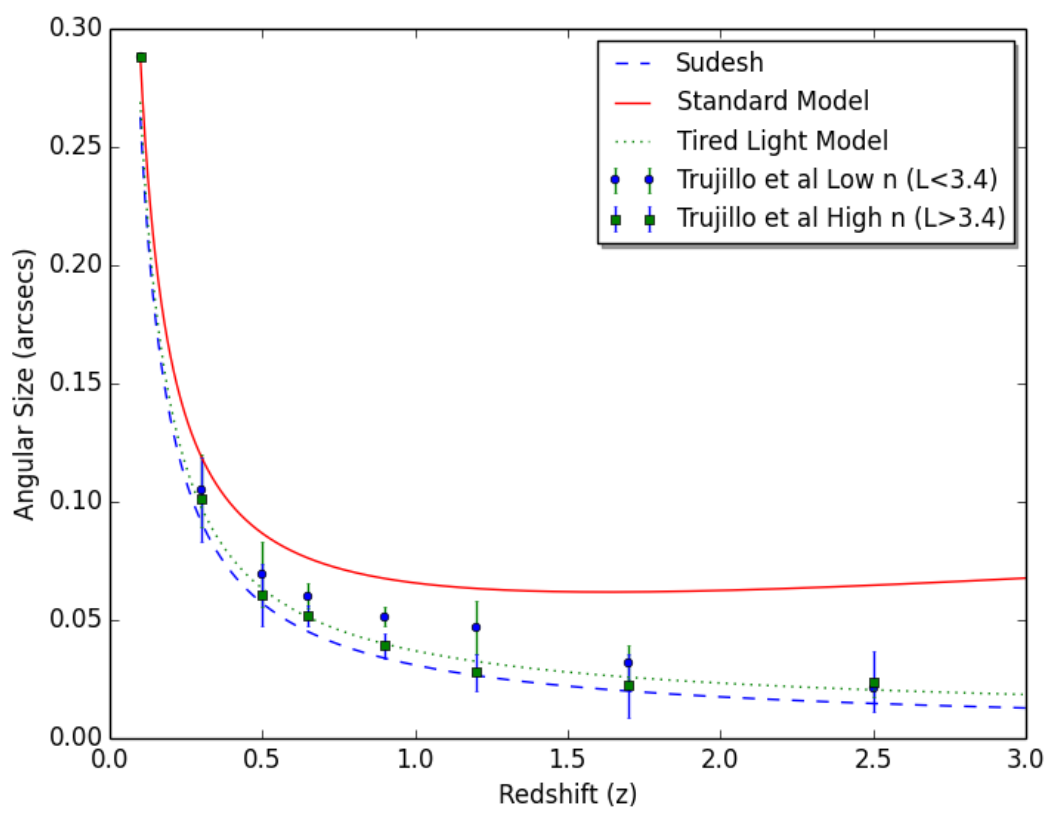

Fig. 4 Angular size versus redshift relation in standard model, tired light model and Metric Contraction model. The actual data points are reverse calculated from standard model theoretical values multiplied by size evolution in look-back time [35]. Both Tired Light model and Metric Contraction model match the observational data without any size evolution while standard model needs high degree of size evolution 